Régis Siqueira de Castro TEIXEIRA ${ }^{1}$

Josué Moura ROMÃO ${ }^{1}$ Suiany Rodrigues CÂMARA ${ }^{1}$ Walber Feijó de OLIVEIRA ${ }^{2}$

Márcia Helena Niza

Ramalho SOBRAL ${ }^{2}$

Adonai Aragão de

SIQUEIRA ${ }^{1}$

William Maciel CARDOSO 2

Correspondência para:

WILLIAMMACIEL CARDOSO

Av. Rogaciano Leite, $200 \mathrm{Apt}^{\circ} 1303 \mathrm{BI}$

Tulipe, Bairro Salinas

60.810-000 - Fortaleza - Ceará

william.maciel@uol.com.br e regissct

@ig.com.br

Recebido para publicação: 17/08/04 Aprovado para publicação: 01/06/2005

\title{
Indução a muda forçada em Galinhas D'Angola (Numida meleagris) através do óxido de zinco
}

\author{
1 - Curso de Medicina Veterinária da Universidade Estadual do Ceará, \\ Fortaleza - CE \\ 2 - Programa de Pós-graduação em Ciências Veterinárias da Universidade \\ Estadual do Ceará, Fortaleza - CE
}

\section{Resumo}

Devido à escassez de estudos sobre muda forçada em aves alternativas de produção, este experimento teve o objetivo de realizar a muda forçada em galinhas D'Angola avaliando as perdas de peso corpóreo (PPC) que promovessem os melhores índices produtivos pós-muda. Com este propósito foram utilizadas 110 galinhas D’angola alojadas individualmente em gaiolas de poedeiras comerciais e, posteriormente, submetidas à muda forçada com 20000 ppm de óxido de zinco na ração. Estas passaram 21 dias recebendo ração e água ad libitum. Para análise da PPC relacionada à produtividade pós-muda foram utilizados 60 aves organizadas nos seguintes grupos: $24 \%(n=18) ; 26 \%(n=18)$; $28 \%(n=12)$ e acima de $30 \%(n=12)$. As outras 50 aves foram sacrificadas para o estudo do aparelho reprodutor, onde se verificou o tamanho e peso do oviduto e peso do ovário com PPC de $0 \%$ e sua regressão à medida que atingiam os níveis de PPC: 24\%;26\%;28\% e acima de 30\%. A média de retorno produtivo foi $60 \%$, sendo o grupo com PPC de $24 \%$ com o melhor índice (100\%), no entanto, este apresentou índice de produção insatisfatório juntamente com o grupo de PPC acima de 30\%. A muda forçada em Galinhas D'Angola foi viável com índices de PPC em torno de $26 \%$ a $28 \%$ e inviáveis com níveis abaixo de $24 \%$ e acima de $30 \%$. Em relação à regressão do aparelho reprodutor, os melhores resultados produtivos foram em torno de $65,15 \%, 90,49 \%$ e $94,27 \%$ para tamanho e peso do oviduto e peso do ovário, respectivamente.

\section{Introdução}

A Galinha D'Angola africana (Numida meleagris) pertence à família Phasianidae, e foi introduzida no Brasil desde a colonização. No entanto, as galinhas D'Angola industriais foram importadas somente na década de 80, entrando no Brasil através da Região Nordeste. Atualmente, o Estado do Ceará é hoje o maior produtor brasileiro. Apesar da boa produtividade na criação da galinha D'Angola em gaiolas, o período produtivo desta é ainda curto. Para prolongá-lo, aplicase à técnica da muda forçada que provoca um descanso completo do aparelho reprodutor, de modo que as aves sofram regressão do ovário e oviduto para rejuvenescimento dos mesmos. Estas condições são imprescindíveis para que as aves obtenham bons rendimentos produtivos durante o ciclo produtivo pós-muda ${ }^{1,2}$. Segundo Miyano ${ }^{3}$ e Cardoso ${ }^{4}$, a ave recupera a sua capacidade reprodutiva com melhoria da qualidade externa dos ovos após a muda forçada obtendo-se assim um novo ciclo de postura.

Vários são os programas citados na literatura ${ }^{5,6,7,8,9}$, capazes de provocar a muda forçada nas aves, dentre eles estão: os métodos nutricionais, em que são oferecidas às aves rações com níveis nutricionais alterados ${ }^{10,11,12,13,14,15,16,19}$ e os métodos clássicos ou de manejo, onde as aves são privadas de alimentos por um período não superior a 10 
dias. A maioria dos métodos utilizados no Brasil e em outros países trata-se de métodos agressivos ao bem estar animal, sendo proibidos pela Sociedade Protetora dos Animais. Segundo Berry ${ }^{16}$ a indução das poedeiras à muda forçada utilizando ração comercial com níveis de 20000 à 25000 ppm de óxido de zinco, constitui o único método alternativo aos métodos agressivos para as aves. Este método não provoca estresse nas aves, pois neste caso não há supressão de alimento e promove a perda de peso corpóreo (PPC) desejada, não havendo grandes mortalidades, além de trazer ao plantel uma produção de ovos mais rápida após a muda forçada. A técnica de muda forçada é aplicada em galinhas há algumas décadas, no entanto a sua utilização em galinha D'Angola não é usual, tornando-se raras as publicações científicas, desta forma a base literária para esta pesquisa são dados publicados para galinhas poedeiras comerciais. Este trabalho visa analisar quais níveis de PPC promovem uma regressão de ovário e oviduto, que possibilitem o melhor rendimento produtivo pós-muda em galinha D'Angola, através do método alternativo de óxido de zinco.

\section{Material e Métodos}

O experimento foi realizado no Laboratório de Estudos Ornitológicos da Faculdade de Veterinária da Universidade Estadual do Ceará. Foram alojadas 110 galinhas D'Angola de linhagens melhoradas na França, das quais 10,40 e 60 formaram o grupo controle, o grupo para análise de regressão e o grupo para a análise produtiva pós-muda, respectivamente. Essas aves se encontravam no final do primeiro ciclo de produção com 70 semanas de idade e foram alojados em gaiolas individuais tipos postura comercial medindo $25,5 \times 44,5 \times 39 \mathrm{~cm}$. As aves foram pesadas, identificadas individualmente através de anilhas numeradas e induzidas à muda forçada utilizando o método proposto por Scotte; Creger ${ }^{17}$, onde adicionou-se 20.000 ppm de óxido de zinco na ração de postura comercial. Durante o tratamento a água foi fornecida "ad libitum" e utilizou-se iluminação natural. O tratamento ocorreu desde o primeiro dia de oferta de óxido de zinco até o período em que as aves atingiram as PPC préestabelecidas de $24 \%$, 26\%, $28 \%$ e acima de $30 \%$, sendo finalizado no vigésimo primeiro dia após o início do tratamento. Dez aves de cada nível de PPC, pertencentes ao grupo de análise de regressão, foram sacrificadas para a extração do ovário e oviduto com o objetivo de analisar a sua regressão. Para análise e comparação da regressão média do ovário e oviduto, um grupo controle com $0 \%$ PPC foi formado antes do início do tratamento, onde 10 aves foram sacrificadas para a mensuração do peso do ovário e oviduto e tamanho dos ovidutos. Os níveis de regressão dos ovidutos foram mensurados através da análise dos tamanhos e pesos obtidos em cada grupo, enquanto para os ovários apenas a variável peso foi considerada. Os níveis de PPC obtidos foram comparados com o grupo controle. Para análise de produção, 60 aves foram selecionadas, sendo estas divididas em quatro grupos de diferentes níveis de PPC com quantidades que variavam de acordo com o número de aves existentes para cada grupo no final do tratamento. Os grupos obtidos foram: PPC 24\% $(n=18)$, PPC $26 \%(n=18)$, PPC $28 \%$ $(n=12)$, PPC acima de 30\% $(n=12)$. Durante 11 semanas pós-muda, foram controlados os parâmetros: mortalidade, número de aves com retorno à produção e percentual de produtividade de cada grupo, sendo este último avaliado semanalmente. A avaliação da regressão do aparelho reprodutor foi realizada a partir da correlação entre as medições de ovários e ovidutos do grupo de análise de regressão com os resultados produtivos do grupo de análise produtiva pós-muda. Para análise estatística foi utilizada a análise de variância e as médias analisadas através do teste de Tukey com $\mathrm{p}<0,05$.

\section{Resultados e Discussões}

A Tabela 1 mostra os níveis médios de perda de peso corpóreo (PPC), a média de peso das aves no início da muda, no final 
deste e também o peso no primeiro dia de postura pós-muda. Podemos observar que quase todos os grupos apresentaram níveis de pesos médios ao primeiro ovo pós-muda inferiores ao peso inicial, exceto o grupo das aves com PPC acima de 30\%.

Os grupos de aves com 28 e acima $30 \%$ de PPC obtiveram os piores índices de mortalidade, no entanto, o primeiro obteve a segunda melhor produção e o último obteve a pior produção. A baixa produtividade, obtida por aves com PPC acima de $30 \%$, pode ser explicada pelo fato de que estas perderam pouco peso e obtiveram um acentuado ganho de peso ao reinicio de postura (Tabela 1). Leeson; Summers ${ }^{13}$, afirmam que aves com pesos elevados provocam efeito negativo sobre a intensidade de postura. Baker et al. ${ }^{2}$, verificaram que os melhores resultados para a produção de ovos de galinhas foram obtidos com a PPC de aproximadamente $28 \%$ durante a muda forçada. Em nosso experimento, resultados semelhantes foram encontrados em galinhas d'Angola, pois o grupo que obteve uma PPC de $26 \%$ obteve os melhores índices produtivos, seguidos do grupo que obteve 28\% de PPC. Desta forma, esses grupos provocaram as melhores PPC, podendo ser considerados os melhores indicadores para a indução da muda forçada em galinhas d'Angola, pois foram às únicas que apresentaram produção durante as 11 semanas avaliadas e sempre com índices de postura acima da média (gráfico 2).

Os resultados do percentual de produtividade pós-muda por diferentes níveis de PPC estão representados no Gráfico 1 , onde podemos observar que os melhores níveis de PPC são os próximos a $26 \%$, seguido de $28 \%$, não diferindo significativamente, sendo que os grupos que possuíram níveis de PPC de 24\% e os níveis superiores a 30\% apresentaram baixos níveis produtivos. Cardoso ${ }^{7}$ relata que as poedeiras comerciais que sofreram PPC de 25\% durante a muda tem maior intensidade de postura nos ciclos pós-muda que as aves que sofreram PPC de $20 \%$.

$\mathrm{Na}$ Tabela 2, podemos observar os índices de retorno produtivo e mortalidade pós-muda geral e divida por grupos. Após a indução a muda com óxido de zinco, $60 \%$ das aves retornam a produção e somente $10 \%$ não retornaram, sendo que $30 \%$ morreram durante o período pós-muda. A maior taxa de mortalidade foi dos grupos que possuíam PPC de $28 \%$ e acima de $30 \%$, ambos com de $50 \%$ de mortalidade. Setenta por cento das aves remanescentes, somente as aves do grupo com PPC de $24 \%$, com percentual que representa $33,3 \%$ no grupo, não retornaram a produção, sendo que todas as outras aves dos diversos grupos entraram em produção. Os grupos que obtiveram os melhores índices de retorno produtivo foram os de PPC de $24 \%$ e $26 \%$, ambos com índices de 66,6\% das aves que foram induzidas a muda forçada.

As aves com PPC de $28 \%$, apesar de obterem boas produções individuais, iniciaram a postura tardiamente (semana 5), mantendo a produção inferior a do grupo

Tabela 1 - Níveis de perda de peso corpóreo de fêmeas de galinhas d'Angola: no início, no final da muda forçada eno primeiro dia de postura pós-muda

\begin{tabular}{cccccc}
\hline $\begin{array}{c}\text { Grupo por } \\
\text { níveis de } \\
\text { PPC (\%) }\end{array}$ & $\begin{array}{c}N^{\circ} \text { de } \\
\text { aves } \\
\text { Iniciais }\end{array}$ & $\begin{array}{c}\text { Peso médio pré- } \\
\text { muda (g) }\end{array}$ & $\begin{array}{c}\text { Peso médio pós- } \\
\text { muda (g) }\end{array}$ & $\begin{array}{c}\text { Peso Ave } \\
1^{\circ} \text { Ovo (g) }\end{array}$ & $\begin{array}{c}\text { Relação entre } \\
\text { peso ao } 1^{\circ} \\
\text { ovo vs. peso } \\
\text { inicial }(\%)\end{array}$ \\
\hline 24 & 18 & 2553 & 1953 & 2260 & $-11,47$ \\
26 & 18 & 2660 & 1970 & 2320 & $-12,78$ \\
28 & 12 & 2350 & 1680 & 1900 & $-19,14$ \\
Ac. 30 & 12 & 2145 & 1475 & 2540 & $+18,41$ \\
\hline
\end{tabular}

Ac. 30 = acima de $30 ; \mathrm{PPC}=$ perda de peso corpóreo 
Tabela 2 - Mortalidade por grupos e porcentagem de aves com retorno produtivo

\begin{tabular}{|c|c|c|c|c|c|c|c|c|c|}
\hline \multirow{2}{*}{$\begin{array}{c}\text { Grupo de } \\
\text { PPC } \\
\%\end{array}$} & \multirow{2}{*}{$\begin{array}{c}\text { Aves } \\
\text { iniciais } \\
n\end{array}$} & \multicolumn{2}{|c|}{ Mortalidade } & \multicolumn{2}{|c|}{$\begin{array}{c}\text { Aves } \\
\text { remanescentes } \\
\text { para } \\
\text { observação } \\
\text { produtiva }\end{array}$} & \multicolumn{2}{|c|}{$\begin{array}{c}\text { Aves vivas que } \\
\text { não } \\
\text { retornaram a } \\
\text { produção }\end{array}$} & \multicolumn{2}{|c|}{$\begin{array}{l}\text { Total geral de } \\
\text { aves que } \\
\text { retornaram a } \\
\text { produção }\end{array}$} \\
\hline & & $\mathrm{n}$ & $\%$ & $n$ & $\%$ & $N$ & $\%$ & $\mathrm{n}$ & $\%$ \\
\hline 24 & 18 & 0 & 0 & 18 & 100 & 6 & 33,3 & 12 & 66,6 \\
\hline 26 & 18 & 6 & 33,3 & 12 & 66,6 & 0 & 0 & 12 & 66,6 \\
\hline 28 & 12 & 6 & 50 & 6 & 50 & 0 & 0 & 6 & 50 \\
\hline Ac. 30 & 12 & 6 & 50 & 6 & 50 & 0 & 0 & 6 & 50 \\
\hline $\begin{array}{c}\text { Todos os } \\
\text { grupos }\end{array}$ & 60 & 18 & 30 & 42 & 70 & 6 & 10 & 36 & 60 \\
\hline
\end{tabular}

Ac. 30 = acima de $30 ; \mathrm{PPC}=$ perda de peso corpóreo

com PPC de 26\%. Estes resultados foram semelhantes ao do grupo com PPC de $24 \%$ que também manteve produção baixa, apesar de ter iniciado a postura na semana 3. Índices de $24 \%$ de PPC apesar de apresentarem um bom número de aves retornando a produção $(66,6 \%)$, obteve o segundo pior índice produtivo. Da mesma maneira, níveis de PPC superiores a 30\% não são bons indicadores para realização da muda forçada devido à tardia e baixa produção, com índices de postura quase sempre abaixo da média. Em estudos realizados por Teixeira et a ${ }^{19}$ utilizando 20000 ppm de óxido de zinco em ração para poedeiras vermelhas chegaram à conclusão que entre diversas faixas de peso, os melhores rendimentos produtivos foram obtidos com PPC de $35 \%$ sendo o percentual produtivo de $72 \%$.

Apesar de se obter um bom índice de aves que retornaram a produção, o índice de mortalidade de 30\% foi considerado muito alto de uma maneira geral e altíssimo nos grupos com PPC de $28 \%$ (50\% de mortalidade) e acima de 30\% (50\% de mortalidade), sendo contrario a afirmação de Berry ${ }^{3}$ que em seus estudos concluiu que o método de indução de muda forçada utilizando-se óxido de zinco em galinhas provocava pouca mortalidade e nenhum estresse nas aves.

O Gráfico 2, retrata a produtividade dos quatro níveis de PPC. O grupo com índices de 28\% de PPC alcançou o maior percentual produtivo durante as semanas 6 a 11. O grupo com PPC de $26 \%$ obteve a melhor e mais homogênea produtividade durante todo o período produtivo analisado, enquanto que o grupo com PPC de $24 \%$ apresentou início de produção a partir da terceira semana, obtendo os segundos melhores índices durante as semanas 4 e 5, melhores índices durante a semana 5 , sendo que a partir desta data houve um declínio produtivo acentuado colocando-o entre as piores produções de todos os grupos até o final da análise produtiva. O grupo com PPC acima de 30\% obteve retorno produtivo tardio, durante a semana 7, com produções acima da média apenas na semana 9 e 11.

A Tabela 3 nos informa a regressão média do aparelho reprodutor dos quatros grupos destinados ao sacrifício para coleta de ovário e oviduto. De acordo com os resultados produtivos, as melhores regressões segundo o comprimento do oviduto foram de $65,16 \%$ para os grupos com PPC de $26 \%$ e de $69,03 \%$ para os grupos com PPC de $28 \%$, onde diferiram significativamente $(p<0,05)$. Os resultados relativos ao peso do ovário indicaram que a melhor perda foi a de $94,27 \%$, seguido a de $96,60 \%$ nos grupos com percentuais de PPC de $26 \%$ e $28 \%$, respectivamente. Para o peso 
Tabela 3 - Tamanho, peso e níveis de regressão do aparelho reprodutor e produção de ovos pós-muda

\begin{tabular}{|c|c|c|c|c|c|c|c|c|}
\hline PPC & \multicolumn{2}{|c|}{ Oviduto } & \multicolumn{2}{|c|}{ Ovário } & \multicolumn{2}{|c|}{ Oviduto } & \multicolumn{2}{|c|}{ Produção } \\
\hline$(\%)$ & $\begin{array}{c}\text { Tamanh } \\
\text { o (cm) }\end{array}$ & $\begin{array}{c}\text { Regressã } \\
O \\
(\%)\end{array}$ & $\begin{array}{l}\text { Peso } \\
\text { (g) }\end{array}$ & $\begin{array}{c}\text { Regressã } \\
0 \\
(\%)\end{array}$ & $\begin{array}{c}\text { Peso } \\
\text { (g) }\end{array}$ & $\begin{array}{c}\text { Regressão } \\
(\%)\end{array}$ & $\begin{array}{l}\text { Ovos } \\
\text { médios } \\
\text { por ave }\end{array}$ & $(\%)$ \\
\hline 24 & $22,873^{a}$ & 58,18 & $3,3178^{a}$ & 93,58 & $\begin{array}{c}4,7335 \\
\text { a }\end{array}$ & 87,64 & $19,250^{a}$ & 25,0 \\
\hline 26 & $19,060^{b}$ & 65,16 & $\begin{array}{c}2,9636 \\
a b\end{array}$ & 94,27 & $\begin{array}{c}3,6409 \\
b\end{array}$ & 90,49 & $35,500^{b}$ & 46,1 \\
\hline 28 & $16,943^{c}$ & 69,03 & $1,7591^{b}$ & 96,60 & $\begin{array}{c}3,4554 \\
b\end{array}$ & 90,98 & $32,833^{b}$ & 42,6 \\
\hline $\begin{array}{l}\text { Ac. } \\
30\end{array}$ & $\begin{array}{c}18,940 \\
\text { bc }\end{array}$ & 65,37 & $\begin{array}{c}2,3483 \\
a b\end{array}$ & 95,46 & $\begin{array}{c}3,5276 \\
b\end{array}$ & 90,79 & $14,000^{a}$ & 18,2 \\
\hline
\end{tabular}

Médias seguidas de letras diferentes na mesma coluna são estatisticamente diferentes pelo teste de Tukey $(p<0,05)$ Ac. 30 = acima de 30; $\mathrm{PPC}=$ perda de peso corpóreo

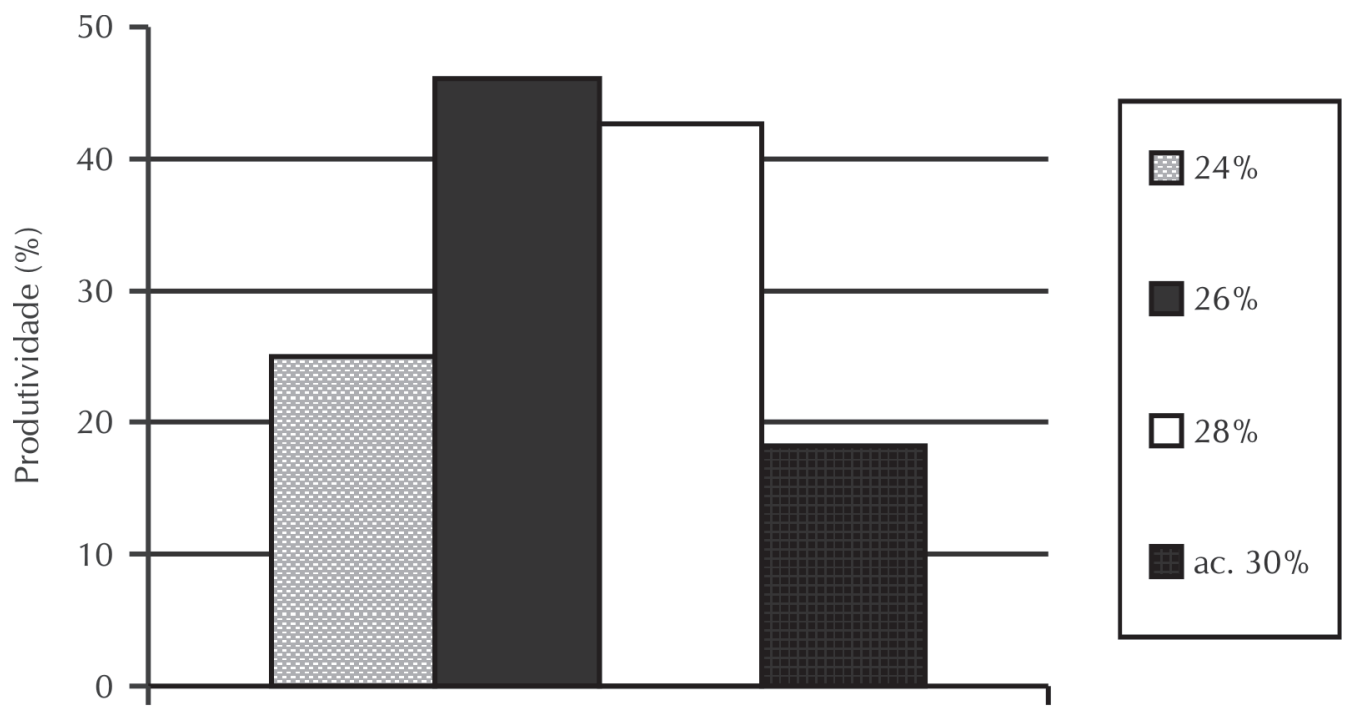

Níveis de perda de peso corpóreo

Figura 1 - Percentual de produtividade pós muda por diferentes níveis de pérda de peso corpóreo

Valores numéricos do Gráfico 1.

\begin{tabular}{cc}
\hline PPC & \% de produção \\
\hline 24 & 25 \\
26 & 46,1039 \\
28 & 42,63636 \\
\hline $30 \%$ & 18,18182 \\
\hline
\end{tabular}

do oviduto, as regressões de 90,49\% e $90,98 \%$ foram as que resultaram em melhores índices produtivos nos grupos de PPC de $26 \%$ e $28 \%$, respectivamente, não havendo diferença significativa. Welty ${ }^{20}$ observou que galinhas fora de produção possuem o peso de oviduto $20 \%$ menor que as de aves em produção. Em nosso experimento as aves 


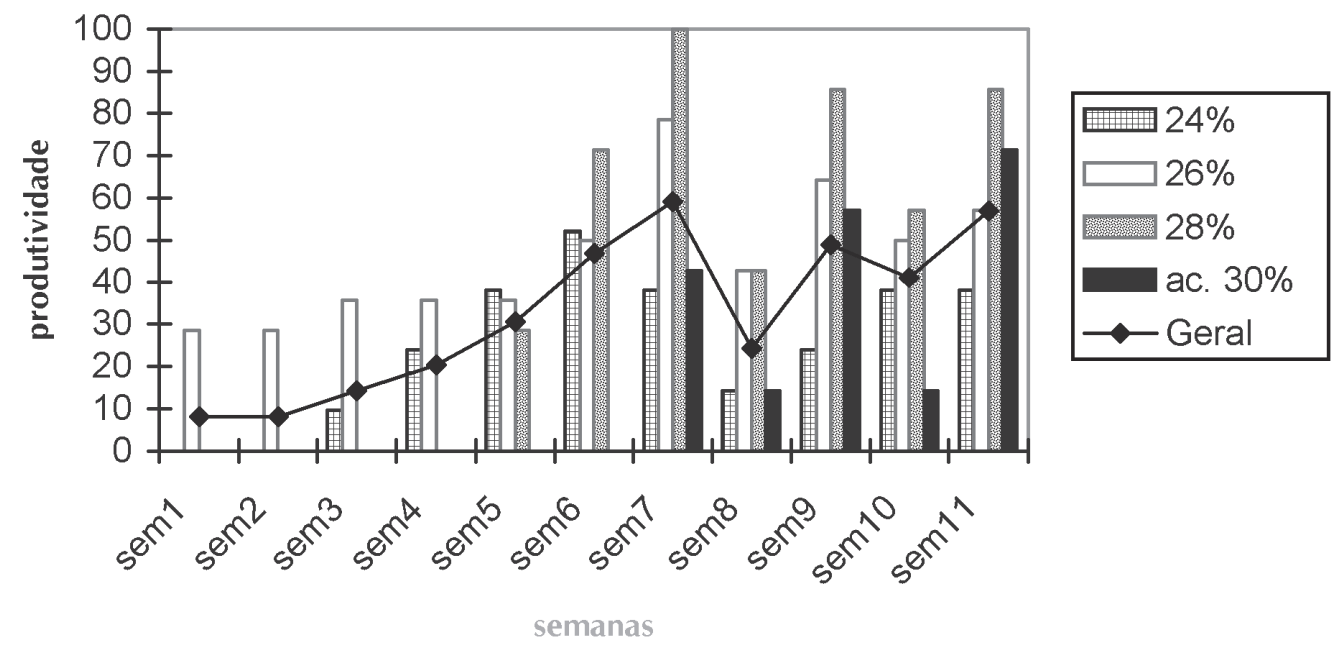

Figura 2 - Produtividade de diferentes níveis de perda de peso vivo durante 11 semanas

Valores numéricos do Gráfico 2

\begin{tabular}{llllllllllll}
\hline Grupo & sem1 & sem2 & Sem3 & sem4 & sem5 & sem6 & sem7 & sem8 & sem9 & sem10 & sem11 \\
\hline Geral & $\mathbf{8 , 1}$ & $\mathbf{8 , 1}$ & $\mathbf{1 4 , 2}$ & $\mathbf{2 0 , 4}$ & 30,6 & 46,9 & 59,1 & 24,2 & 49 & 41 & 57 \\
& & & & & & & & & & & \\
$24 \%$ & 0 & 0 & 9,5 & 23,8 & 38 & 52,3 & 38 & 14,2 & 23,8 & 38 & 38 \\
$26 \%$ & 28,5 & 28,5 & 35,7 & 35,7 & 35,7 & 50 & 78,5 & 42,8 & 64,2 & 50 & 57,1 \\
$28 \%$ & 0 & 0 & 0 & 0 & 28,5 & 71,4 & 100 & 42,8 & 85,7 & 57,1 & 85,7 \\
\hline $\begin{array}{l}\text { ac. } \\
30 \%\end{array}$ & 0 & 0 & 0 & 0 & 0 & 0 & 42,8 & 14,2 & 57,1 & 14,2 & 71,4 \\
\hline
\end{tabular}

do grupo com PPC de $28 \%$ possuíram regressão média de até 90,98\% de seu peso inicial. Em geral, os resultados de regressão obtidos estão de acordo com Woltford ${ }^{22}$ que analisando vários métodos de indução a muda verificou redução de ovário e oviduto, havendo portanto uma relação direta entre a PPC e a redução do aparelho reprodutor. De uma maneira geral, em nosso experimento, foi obtido resultado de regressões superiores aos encontrado por Koelkebeck ${ }^{12}$, onde trabalhando com poedeiras brancas, obteve resultados em que o ovário representava $0,2 \%$ do peso corpóreo, já em nosso trabalho todas as aves obtiveram maiores regressões. $\mathrm{O}$ mesmo aconteceu com relação ao oviduto em que todas as aves obtiveram seus órgãos representando pesos menores a $0,5 \%$ do peso corpóreo.

\section{Conclusões}

Podemos concluir que a muda forçada em capotes é viável com índices de perda de peso corpóreo em torno de $26 \%$. Níveis abaixo de $24 \%$ e acima de $30 \%$ não são recomendáveis para a realização de um programa de muda forçada através do método do óxido de zinco. Em relação a variável regressão do aparelho reprodutor, obtêm-se os melhores resultados produtivos com regressões em torno de $65,15 \%, 94,27 \%$ e $90,49 \%$ para tamanho do oviduto, peso do ovário e peso do oviduto, respectiva-mente. 


\title{
Induced molt in Guinea fowls (Numida meleagris) through the use of zinc oxide
}

\begin{abstract}
Due to scarcity of studies on forced molt in alternative production birds, this experiment had the objective of performing an induced molt in Guinea Fowls observing the best body weight losses (BWL) that could promote good post-molt productions. With this purpose, 110 Guinea fowls were housed in individual commercial cages and were submitted to induced molt through the addition of zinc oxide in feed with the concentration of 20000 ppm during 21 days and received drinking water ad libitum. In the analysis of BWL related to post-molt productivity, 60 birds were organized in the groups, according to the percentage of BWL: $24 \%(n=18), 26 \%(n=18), 28 \%$ $(n=12)$ and more than $30 \%(n=12)$. The other 50 birds were sacrificed for analyses of the reproductive organs, in which the oviduct size and weight of oviduct and ovary were measured with $0 \%$ of BWL and since then the regression of these organs were calculated to $24 \%$, $26 \%, 28 \%$ and more than $30 \%$ of BWL. The medium of post-molt return to production was $60 \%$ and the group of $24 \%$ of BWL presented the best productive return $(100 \%)$, however this group presented an insufficient production rate as the birds with $30 \%$ of BWL. In this way, the induced molt in Guinea fowls was viable with BWL around 26 and $28 \%$ and unviable with less than $24 \%$ or more than $30 \%$. In relation to regression of the reproductive organs the best productive results were $65,15 \%, 90,49 \%$ and $94,27 \%$ to oviduct size and weight and ovary weight, respectively.
\end{abstract}

\section{Referências}

1 BAKER, M.; BRAKE, J.; MCDANIEL, G. R. The relationship between body weight loss during a forced molt and post-molt reproductive performance of caged layers. Poultry Science, v. 60, p. 1594, 1982.

2 BAKER, M.; BRAKE, J.; MCDANIEL, G. R. The relationship between body weight loss during an induced molt and postmolt egg production, egg weight, and shell quality in caged layers. Poultry Science, v. 62, p. 409-413, 1983.

3 MIYANO, O. A. Viabilidade econômica da muda forçada em poedeiras comerciais. In: CONFERÊNCIA APINCO DE CIÊNCIA E TECNOLOGIA AVÍCOLAS, Santos, SP. Anais. Campinas: FACTA, p. 159-166, 1993.

4 CARDOSO, W. M. Muda forzada de ponedores comerciales: influencia de la perdida de peso vivo sobre las principales variables productivas y de calidad fisica del huevo. 1996. Thesis (Doctoral)-Escuela Técnica Superior de Ingieneros Agronomos, 1996.

5 BERRY, W. D.; BRAKE, J. Comparison of parameters associated with molt induced by fasting, zinc and low dietary sodium in caged layers. Poultry Science, v. 64 , p. 2027-2036, 1985.
Key-words: Forced Molt. Guinea Fowls. Ovary. Oviduct. Zinc Oxide.
6 HUSSEIN, A. S.; CANTOR, A. H.; JOHNSON, T. H. Use of high levels of dietary aluminun and zinc for inducing pauses in egg production of Japonese quail. Poultry Science. v. 67, p. 1157-1165, 1988.

7 HUSSEIN, A. S. Induced moulting procedures in laying fowl. World's Poultry Science. J., v. 52. p. 175-187, 1996.

8 HARMS, R. S. Effect of removing salt sodium or chloride from the diet of commercial layers. Poultry Science. v. 70; p, 333-336, 1991.

9 BREEDING, S. W.; BRAKE, J.; GARLICK, J. D. Moulted induced by dietary zinc in a loss calcium diet. Poultry Science, v. 71, p. 168-180, 1992.

10 HURWITZ, S.; BORNSTEIN, S.; LEV, Y. Some responses of laying hens to induced arrest of egg production. Poultry Science. v. 54, p. 415-422, 1975.

11 WHITEHEAD, C. C.; SHANNON, D. W. F. The control of egg production using a low-sodium diet. British Poultry Science, v.15, p. 429-434, 1974.

12 SAID, N. W. et al. comparison of the effect of two forced molting methods on performance of two commercial strains of laying hens. Poultry Science, v. 63, p. 2399-2403, 1984.

13 CANTOR, A. H.; JOHNSON, T. H. Inducing pauses 
in egg production of Japonese quail with dietary zinc. Poultry Science.v. 63, p. 10, 1984 Suplementum.

14 MCORMICK, C. C.;CUNNINGHAM, D. L. High dietary zinc and fasting as methods of forced resting: a performance comparision. Poultry Science, v.63, p. 1201-1206, 1984.

15 MCCORMICK, C. C.; CUNNINGHAM,D. L. Performance and physiological profiles of high dietary zinc and fasting as methods of inducing a forced rest. A direct comparision. Poultry Science., v. 66, p. 10071013, 1987

16 BERRY, W. D. A physiological comparison of methods for induced molting in the laying hen. Thesis (M. S.) North Caroline State University, Raleigh, 1984.

17 SCOTT, J. T.; CREGER, C. R. The use of zinc as an effective molting agent in laying hens. Poultry Science, v. 55 , p. $2089,1976,1976$.

18 LEESON, S.; SUMMERS, J. D. Effect of immature body weight on laying performance. Poultry Science, v. 66, p. 1921-1928, 1987.

19 TEIXEIRA, R. S . C. et al. Relação entre níveis de perda de peso corpo com a regressão de ovário e oviduto em poedeiras semipesadas submetida ao processo de muda forçada com oxido de zinco. Ciência Animal, v. 12, p. 99-101, 2002.

20 WELTY J. C. The life of birds. Sondon: W. B. Saunders, 1962.

21 WOLFORD, J. H. Induced molting in laying fowls. Poultry Science Journal, v. 40, p. 60-73, 1984.

22 KOLKEBECK, K. W.; PARSCNS, C. M. Evaluation of non-feed vs fees withdrawal methods for induced molting of laying hens. (A Report submitted to the United Egg Producers and Ridley Feed Ingredients). Department of Animal Sciences University of Illinois Urbana, Illinois 61801. Disponível em: <http:// www.ridleyfeedingredients.com/moltingstudy.pdf $>$. Acesso em 04 de agosto de 2004. 